\title{
Decay of the toroidal field in magnetically driven jets
}

\author{
R. Moll \\ Max-Planck-Institut für Astrophysik, Karl-Schwarzschild-Str. 1, 85748 Garching, Germany \\ e-mail: rmo@mpa-garching.mpg.de
}

Received 3 April 2009 / Accepted 4 July 2009

\section{ABSTRACT}

\begin{abstract}
A 3D simulation of a non-relativistic, magnetically driven jet propagating in a stratified atmosphere is presented, covering about three decades in distance and two decades in sideways expansion. The simulation captures the jet acceleration through the critical surfaces and the development of (kink-)instabilities driven by the free energy in the toroidal magnetic field component. The instabilities destroy the ordered helical structure of the magnetic field, dissipating the toroidal field energy on a length scale of about 2-15 times the Alfvén distance. We compare the results with a 2.5D (axisymmetric) simulation, which does not become unstable. The acceleration of the flow is found to be quite similar in both cases, but the mechanisms of acceleration differ. In the 2.5D case approximately $20 \%$ of the Poynting flux remains in the flow, in the 3D case this fraction is largely dissipated internally. Half of the dissipated energy is available for light emission; the resulting radiation would produce structures resembling those seen in protostellar jets.
\end{abstract}

Key words. magnetohydrodynamics (MHD) - ISM: jets and outflows - ISM: Herbig-Haro objects - galaxies: jets gamma rays: bursts

\section{Introduction and rationale of the calculations}

A magnetized outflow produced by a rotating magnetic object has become the default interpretation for objects ranging from protostellar jets to gamma-ray bursts. The outflow in this model contains a tightly wound helical magnetic field. Such a nearly toroidal field represents a source of free energy that makes the flow inherently prone to non-axisymmetric magnetic instabilities. In this study we investigate the longer-term development of such instabilities and their consequences for jet phenomenology.

Instabilities are not necessarily fatal for the jet. Kink instabilities of helical magnetic field configurations typically saturate at a finite amplitude. Such instabilities at moderate amplitudes have been invoked to explain phenomena like the wiggly appearance of Herbig-Haro objects (Todo et al. 1993) or the orientation of VLBI jets in AGN (Königl \& Choudhuri 1985).

The development of kink instability in a jet is not the same as in a laboratory configuration. Due to the sideways expansion of the flow, the ratio of poloidal (stabilizing) to toroidal (destabilizing) field components decreases with distance along the flow. Conditions favorable for instability are thus continually recreated in such a flow. In Moll et al. (2008, hereafter Paper I), we presented 3D MHD simulations showing the onset of instabilities in an expanding jet created by twisting a purely radial magnetic field. The degree of instability was found to depend on the kind of rotation that generates the twist. The highest degree of instability was attained with a constant angular velocity (rigid rotation); the jet produced in this case was subject to helical deformations with large amplitudes, causing sideways displacements of several degrees. However, the magnetic structure of the jet was not disrupted within the computational volume, and the instability did not lead to a significant decrease of the Poynting flux. These results indicate the need to follow the instabilities to larger distances from the source. We present here the results of simulations extending to a distance of 1000 times the diameter of the jet source.

In Paper I we showed how the degree of instability depends on the way in which the jet is collimated by its environment. If collimation conditions are such that the opening angle of the jet increases with distance, the Alfvén travel time across the jet increases more rapidly than the expansion time scale. As a result, instabilities soon "freeze out", and decay of the toroidal field becomes ineffective. In better collimating jets, such that the opening angle narrows with distance, instability is always effective. The calculations presented here are for such a case. It is probably the most relevant for both AGN and protostellar jets. Observations of the jets in M 87 (Junor et al. 1999) and HH30 (Mundt et al. 1990), for example, show a rapidly decreasing opening angle in the inner regions of the jet.

The dissipation of the magnetic energy in the toroidal field heats the plasma. Radiation produced by this plasma would be an alternative or complement to the standard mechanism invoked, which relies on dissipation in internal shocks. Observations such as those of M 87 and HH30 indicate that dissipation starts fairly close to the central object, compared with most observable length scales, but also that the innermost regions, perhaps comparable to the Alfvén distance $r_{\mathrm{A}}$, are quiet. Inferences from VLBI observations indicate that AGN jets are not magnetically dominated on most observable scales (cf. Sikora et al. 2005, and references therein); decay of the magnetic field relatively close to the source of the jet would fit this observation (see also discussion in Giannios \& Spruit 2006).

If dissipation takes place close enough to the source, it is possible that it can be studied realistically by $3 \mathrm{D}$ simulations with current computational resources. In Paper I, we already found indications that decay of the toroidal field may become important at distances as close as 10-30 times $r_{\mathrm{A}}$.

The release of magnetic energy by the instability may also be important for accelerating the flow (Drenkhahn 2002). 
Dissipation of toroidal field causes the magnetic pressure gradient along the jet to steepen; this adds an accelerating force that is absent when the toroidal field is conserved (Giannios \& Spruit 2006; Spruit 2008).

To facilitate extraction of physics from the numerical results, the 3D results in the following are compared systematically with a $2.5 \mathrm{D}$ (axisymmetric) simulation corresponding to the same initial and boundary conditions.

\section{Methods}

\subsection{Numerical MHD solver, grid and coordinates}

The following is a brief summary; for details see Paper I where the same numerical approach but different initial conditions were used. Details on the MHD code can also be found in Obergaulinger (2008).

We numerically solve the ideal adiabatic MHD equations in a static external gravitational potential $\Phi \propto r^{-1}$ on a spherical grid $(r, \theta, \phi)$. In the $2.5 \mathrm{D}$, axisymmetric simulation, the jet propagates along the coordinate axis $\theta=0$. In the 3D simulation, the jet's axis is taken in the direction $\theta=\phi=\pi / 2$. The jet thus propagates in equatorial direction of the coordinate system. This avoids the coordinate singularity along the polar axis $(\theta=0)$, which is numerically problematic for trans-axial flows such as are caused by instabilities. The computational volume covers a range $\Delta \theta=\Delta \phi$ that comprises about twice the expected opening angle of the jet. The spacing of the computational grid is uniform in the angular directions and logarithmic in the radial direction. In this way, the varying numerical resolution approximately matches the increase of the natural length scales in the expanding jet.

For presentation and discussion, we transform the results to a different coordinate system. This is again a spherical coordinate system, but with the polar axis aligned with the jet. The polar and azimuthal angles in this system are denoted by $\vartheta$ and $\varphi$, respectively:

$$
\begin{aligned}
& \sin ^{2} \vartheta=1-\sin ^{2} \theta \sin ^{2} \phi, \\
& \tan \varphi=\tan \theta \cos \phi
\end{aligned}
$$

in the $3 \mathrm{D}$ simulation and $\vartheta \equiv \theta, \varphi \equiv \phi$ in the $2.5 \mathrm{D}$ simulation. $R:=r \sin \vartheta$ is used to denote the distance to the axis (cylindrical radius).

Dissipation of magnetic energy in the flow causes heating. In nature this would lead to losses by radiation; in the computations it can cause numerical problems in regions where the magnetic energy dominates. Instead of a more realistic model for such losses, a temperature-control term is added in the energy equation, such that the temperature relaxes to that of the initial state on an appropriate time scale. In the simulations presented here this time scale is chosen such that the temperature stays within about a factor 100 around the initial value.

\subsection{Initial and boundary conditions}

The initial state consists of a current-free magnetic field embedded in a stratified atmosphere in the gravitational field of a point mass. The field configuration of this initial state is of a "collimating" type, the distance between neighboring field lines increases less rapidly than the distance from the source $r$. In Paper I, we showed that instabilities develop more strongly in such collimating configurations than in a purely radial initial field.

\subsubsection{Initial field configuration}

The initial field is axisymmetric around the jet axis and hence can be written as

$\boldsymbol{B}=\frac{1}{R} \boldsymbol{\nabla} \psi \times \hat{\boldsymbol{e}}_{\varphi}=\boldsymbol{\nabla} \times\left(\frac{1}{R} \psi \hat{\boldsymbol{e}}_{\varphi}\right)=\boldsymbol{\nabla} \times \boldsymbol{A}$,

where $\psi$ is the stream function of the field. We construct the initial condition as a linear combination of the stream function of a monopole field, given by

$\psi_{\text {mono }} \propto 1-\cos \vartheta$

and a field with the stream function

$\psi_{\text {para }} \propto \sqrt{\left(\frac{R}{R_{0}}\right)^{2}+\left(1+\frac{z}{R_{0}}\right)^{2}}-\left(1+\frac{z}{R_{0}}\right)$,

$(z>0)$ of which the field lines have a parabolic shape (Cao \& Spruit 1994). Here, $z=r \cos \vartheta$ denotes the height along the jet's central axis (which is actually the $y$-axis in the $3 \mathrm{D}$ simulation and the $z$-axis in the $2.5 \mathrm{D}$ simulation). The weighting of the two components is parametrized with the quantity

$\zeta:=\left.\frac{B_{\text {mono }}}{B_{\text {para }}}\right|_{r=r_{\mathrm{b}}, \vartheta=0}$,

the relative strength of the constituent fields at the lower boundary $r=r_{\mathrm{b}} . \zeta \rightarrow \infty$ corresponds to a pure monopole field and $\zeta=0$ corresponds to a pure parabolic field. The radius $r_{\text {equ }}$ at which $B_{\text {mono }}=B_{\text {para }}$ on the central axis follows from

$\zeta=\frac{r_{\text {equ }}^{2}\left(R_{0}+r_{n}\right)}{r_{n}^{2}\left(R_{0}+r_{\text {equ }}\right)}$.

The lower the value of $\zeta$ or $r_{\text {equ }}$, the more collimating is the shape of the initial field.

\subsubsection{Stratification}

We impose the static gravitational field of a point mass, located at the origin of the coordinate system. The stratification is initially in hydrostatic equilibrium in this potential. The gas pressure is chosen such that the plasma- $\beta:=p / p_{\text {mag }}$ in the initial state is approximately constant at small radii $\left(\ll r_{\text {equ }}\right)$, where the monopole field dominates: $p \propto r^{-4}, \rho \propto r^{-3}, T \propto r^{-1}, c_{\mathrm{S}} \propto r^{-1 / 2}$ and $v_{\mathrm{A}} \propto r^{-1 / 2}$ in the initial state. At large radii, the value of the initial $\beta$ is reduced as the magnetic field strength decreases less rapidly than $r^{-2}$.

\subsubsection{Boundaries}

Boundary conditions are maintained through the use of "ghost cells" outside the computational domain. At the sides ( $\theta$ in the 2.5D simulation, $\theta$ and $\phi$ in the 3D simulation) and top (upper $r$ ), we use open boundary conditions that allow for an almost forcefree outflow or inflow of material, including magnetic fields.

The bottom boundary is located at a finite height $r_{\mathrm{b}}$ above the origin of the gravitational potential. This distance is $1 / 200$ of the size of the computational volume. The jet is generated there by a "rotating disk" 1 of radius $R_{\mathrm{b}}$ around the axis. It is implemented by maintaining a velocity field $\boldsymbol{v}=v_{\varphi} \hat{\boldsymbol{e}}_{\varphi}$ in the ghost

\footnotetext{
1 Strictly speaking, because of the spherical grid, what we call "disk" here is really a spherical cap with small curvature.
} 
Table 1. Normalization units.

\begin{tabular}{ccc}
\hline \hline Quantity & Symbol(s) & Unit \\
\hline length & $x, y, z, r, R$ & $l_{0}$ \\
gas pressure & $p$ & $p_{0}$ \\
density & $\rho$ & $\rho_{0}$ \\
mass flow rate & $\mathcal{M}$ & $\rho_{0} l_{0}^{3} / t_{0}$ \\
velocity & $v$ & $c_{\mathrm{s} 0}=\sqrt{\gamma p_{0} / \rho_{0}}$ \\
time & $t$ & $t_{0}=l_{0} / c_{\mathrm{s} 0}$ \\
energy & $E$ & $p_{0} l_{0}^{3}$ \\
energy flow rate & $\mathcal{E}$ & $p_{0} l_{0}^{3} / t_{0}$ \\
force density & $F$ & $p_{0} / l_{0}$ \\
power & $P$ & $P_{0}=p_{0} l_{0}^{3} / t_{0}$ \\
magnetic flux density & $B$ & $B_{0}=\sqrt{8 \pi p_{0}}$ \\
current density & $j$ & $j_{0}=B_{0} c /\left(4 \pi l_{0}\right)$ \\
\hline
\end{tabular}

zones: $v_{\varphi}=v_{\varphi, \mathrm{b}}^{\max } R / R_{\mathrm{b}}$ for $R \leq R_{\mathrm{b}}$ and 0 elsewhere. The disk thus rotates rigidly like in the cases R2 and R3 in Paper I.

All quantities at the bottom boundary except for $\boldsymbol{B}$ are fixed at their initial values in the ghost cells. $\boldsymbol{B}$ is extrapolated from the interior of the domain.

\subsection{Parameters and units}

The above suggests a specification of the problem in terms of 7 parameters: the field configuration parameter $\zeta$, a scale for the field strength, scales for the pressure and density, the bottom boundary location $r_{\mathrm{b}}$, the radius $R_{\mathrm{b}}$ of the rotating disk, and its rotation rate. Because of the symmetries of the problem, it is actually determined by only 4 parameters; the remaining 3 dependences are equivalent to scaling factors. As the 4 independent parameters we choose the following dimensionless quantities: the plasma- $\beta$ of the initial state, the angle $\vartheta_{\mathrm{b}}=\arctan \left(R_{\mathrm{b}} / r_{\mathrm{b}}\right)$ which controls the opening angle of the flow, the field configuration parameter $\zeta$, and finally the Alfvénic Mach number $M_{\mathrm{A}}$ of the rotating velocity field at the edge $\left(R=R_{\mathrm{b}}\right)$ of the launching disk, which controls the power of the jet. They have the values $\beta=1 / 9, \zeta=30, \vartheta_{\mathrm{b}}=5.7^{\circ}, M_{\mathrm{A}}=0.1$. (The simulations reported in Paper I corresponded to $\beta=1 / 9, \zeta \rightarrow \infty, \vartheta_{\mathrm{b}}=5.7^{\circ}$, $M_{\mathrm{A}}=0.1$.)

The units used for reporting the results below are the length scale $l_{0} \equiv 2 R_{\mathrm{b}}$, and the pressure and density on the axis at the bottom boundary, $p_{0} \equiv p_{\mathrm{b}}, \rho_{0} \equiv \rho_{\mathrm{b}}$. Together with the 4 model parameters $\beta, \zeta, \vartheta_{\mathrm{b}}$ and $M_{\mathrm{A}}$, the units for other quantities follow from these as shown in Table 1.

The simulations cover a distance of 2000 times the initial jet radius $R_{\mathrm{b}}$ in the spherical range $5<r<1005$. The resolution used in the $3 \mathrm{D}$ simulation is $768 \times 128 \times 128$; the corresponding domain size is $1000 \times 33.8^{\circ} \times 33.8^{\circ}$. The resolution used in the $2.5 \mathrm{D}$ simulation is $768 \times 96$; the corresponding domain size $1000 \times 16.8^{\circ}$. In both simulations, the radial width $\Delta r$ of the grid cells increases from 0.03 at the lower boundary to 6.92 at the upper boundary in logarithmic steps.

\section{Results}

The 3D simulation was done using MPI parallelization on 128 CPUs, the 2.5D simulation was done with OpenMP parallelization on 32 CPUs.

The jet crossed the upper boundary of the computational volume at the physical time $t \approx 523$ on the 13th wall clock day of the 3D simulation. We stopped it after 26 days, at which time $t=1055$ had been reached. The $2.5 \mathrm{D}$ simulation ran for $24 \mathrm{~h}$, reaching $t=1732$.
The 3D jet is subject to non-axisymmetric instabilities, evidently of the kink $(m=1)$ kind. They have a disruptive effect on the magnetic field structure and cause the toroidal field to decay, see Fig. 1. If such a jet was observed, it would probably look similar to Fig. 2, with bright knots and wiggles being prominent features. The knots move at a substantial fraction of the flow speed, sometimes merging or fading before leaving the computational domain. The $2.5 \mathrm{D}$ jet does not exhibit any form of instability.

\subsection{Acceleration, collimation and mass flow}

Most of the acceleration takes place below $r=100$, where the jet reaches $\gtrsim 80 \%$ of its terminal speed, see Fig. 3. The location of the sonic $\left(v_{r}=c_{\mathrm{s}} \approx c_{\mathrm{s}} v_{\mathrm{A}} /\left(c_{\mathrm{s}}^{2}+v_{\mathrm{A}}^{2}\right)^{1 / 2}\right.$, the slow magnetosonic cusp speed), Alfvén $\left(v_{r}=v_{\mathrm{A} r}\right)$ and fast magnetosonic $\left(v_{r}^{2}=c_{\mathrm{s}}^{2}+v_{\mathrm{A}}^{2}\right)$ radii depends on the direction $\vartheta$, see inset in Fig. 3. This is because the acceleration is more effective near the boundary of the jet, which is a consequence of the rigid rotation profile used in its generation, and because the poloidal magnetic field is being redistributed such that the local Alfvén velocity becomes relatively high near the axis. As the toroidal field energy, which determines the jet acceleration and the development of instabilities, is concentrated towards the jet boundary (as opposed to the axis), the Alfvén radius there is probably the most important for the subsequent considerations.

The central velocity tends to be higher in the $3 \mathrm{D}$ simulation, presumably due to a more effective transfer of momentum from the boundary to the center. This may, together with the entrainment of ambient material discussed below, also explain why the peak velocities are somewhat lower in the 3D simulation.

The instabilities have a noticeable effect on the collimation behavior, see Fig. 4. In the 2.5D simulation, the opening angle of the jet decreases by about $2^{\circ}$ in the first half of the computational volume, which is about $1^{\circ}$ less than what is marked by the shape of the initial magnetic field. In the second half, well beyond the Alfvén surface, the opening angle settles to a constant value. The jet in the $3 \mathrm{D}$ simulation is less collimated ${ }^{2}$, the location of the boundary fluctuates with time in the unstable region. Averaged over time, we find it to be nearly conical.

The mass flow rate $\mathcal{M}(t, r):=\int_{r=\text { const }} \rho v_{r} \mathrm{~d} A$ is somewhat higher in the $3 \mathrm{D}$ simulation and subject to strong fluctuations above $r \approx 200$ : the mean value over all radii at $t \approx 1060$, measured in the unit for $\mathcal{M}$ listed in Table 1 , is $0.068 \pm 8 \%$ in the $2.5 \mathrm{D}$ and $0.082 \pm 34 \%$ in the $3 \mathrm{D}$ simulation. The average over several time steps shows a slight increase of $\mathcal{M}$ with $r$ in the $3 \mathrm{D}$ case, whereas no trend can be deduced in the $2.5 \mathrm{D}$ case. This may be an indicator for an enhanced entrainment of ambient material caused by the instabilities. It may also in part explain the lower peak velocities in the $3 \mathrm{D}$ simulation.

\subsection{Energy}

A comparison of the different kinds of energy flow rates gives information about energy transformations taking place in the jet. Integrating the radial energy flux

$$
\underbrace{\frac{1}{2} \rho v^{2} v_{r}}_{\text {kinetic }}+\underbrace{\frac{\gamma}{\gamma-1} p v_{r}}_{\text {thermal enthalpy }}+\underbrace{\rho \Phi v_{r}}_{\text {grav. potential }}+\underbrace{S_{r}}_{\text {magnetic enthalpy }}
$$

\footnotetext{
2 Note, however, that with our definition of the jet boundary, a rigid displacement away from the center also increases the opening angle.
} 


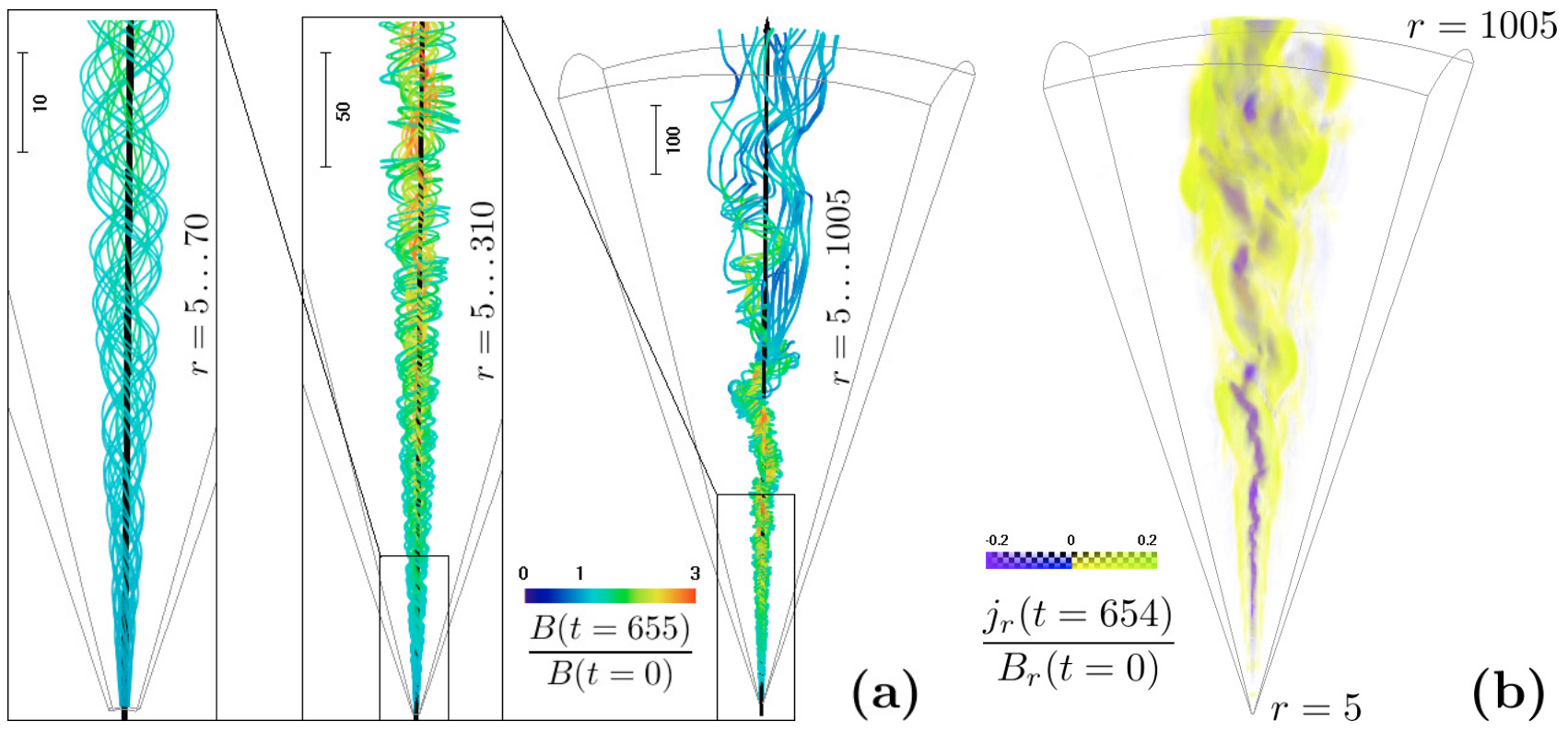

Fig. 1. a) Selected magnetic field lines in the 3D simulation on successively increasing length scales. The color coding gives the magnetic field strength relative to its initial value. The field lines shown are the ones that are anchored in the rotating disk at the lower boundary. The jet starts out with a helical magnetic field (left image) whose toroidal component becomes increasingly stronger (middle image) until instabilities disrupt the ordered structure, the toroidal field decays and the field becomes predominantly poloidal (right image). b) Radial component of the current density $(\boldsymbol{\nabla} \times \boldsymbol{B})$ in the 3D simulation. The backward current, shown in blue here, is concentrated on the central axis until being first displaced and then disrupted by instabilities.

over the $r=$ const. surface, we obtain the rate of energy $\mathcal{E}_{\text {tot }}(r)$ that crosses the surface. The individual components, which we denote with $\mathcal{E}_{\text {kin }}, \mathcal{E}_{\text {thrm }}, \mathcal{E}_{\text {grav }}$ and $\mathcal{E}_{\text {mag }}$, are plotted in Fig. 5. As the values are strongly fluctuating in the unstable region, we averaged over time in the $3 \mathrm{D}$ case. $\mathcal{E}_{\mathrm{mag}}$ is reduced by $80 \%$ in the $2.5 \mathrm{D}$ simulation and by $94 \%$ in the $3 \mathrm{D}$ simulation along the simulated distance, the gap between the curves widens considerably where the $3 \mathrm{D}$ jet exhibits instabilities. We presume this to be caused by (numerical) magnetic dissipation, which turns magnetic energy into heat, causing an increase in $\mathcal{E}_{\text {thrm }}$. The environmental thermal energy is not affected much. As our calculations include an energy loss term (see Sect. 2.1), there is no one-to-one correspondence between the dissipated energy and the increase in $\mathcal{E}_{\text {thrm }}$.

The final contribution of $\mathcal{E}_{\text {mag }}$ to $\mathcal{E}_{\text {tot }}$ is $24 \%$ in the $2.5 \mathrm{D}$ simulation and $9 \%$ in the $3 \mathrm{D}$ simulation. $\mathcal{E}_{\text {kin }}$ is up to about $10 \%$ smaller in the 3D simulation and does not show a dissipationinduced increase. $\mathcal{E}_{\text {kin }}$ arises from azimuthal and radial motion, with the relative share being highly similar in the $2.5 \mathrm{D}$ and 3D case: the azimuthal contribution drops continuously to values $<5 \%$ at $r>200$ and the contribution from $v_{\vartheta}$ is insignificant throughout.

$\mathcal{E}_{\text {mag }}$ can be decomposed further. The radial component of the Poynting vector has 4 terms,

$S_{r}=\frac{1}{4 \pi}\left(B_{\vartheta}^{2} v_{r}+B_{\varphi}^{2} v_{r}-B_{\vartheta} B_{r} v_{\vartheta}-B_{\varphi} B_{r} v_{\varphi}\right)$.

Integrating these terms over the $r=$ const. surface gives the components [in order of their appearance in Eq. (9)] $\mathcal{E}_{\text {mag }}^{1} \ldots \mathcal{E}_{\text {mag }}^{4}$ which, added together, give $\mathcal{E}_{\text {mag }}$. These components of the magnetic enthalpy flow rate are plotted in the lower panel of Fig. 5. At very small radii, the most important term is $\mathcal{E}_{\text {mag }}^{4}$, the work done by the azimuthal flow against the azimuthal component of magnetic stress. Its contribution to $\mathcal{E}_{\text {mag }}$ decreases rapidly, however, falling below $50 \%$ at $r \approx 30$ (i.e. well below the Alfvén surface, near the sonic one). It is then taken over by $\mathcal{E}_{\mathrm{mag}}^{2}$, which describes the flow of magnetic enthalpy stored in the azimuthal field. $\mathcal{E}_{\text {mag }}^{1}$ has minor significance in the 3D simulation, which may be due to the perturbed toroidal field having also a $B_{\vartheta}$ component (a rigid displacement of a pure azimuthal field in the $r=$ const. plane introduces a non-azimuthal component). $\mathcal{E}_{\mathrm{mag}}^{3}$ is insignificant in both cases. The strong decrease of $\mathcal{E}_{\text {mag }}$ in the $3 \mathrm{D}$ simulation is caused by the decrease of $\mathcal{E}_{\mathrm{mag}}^{2}$.

We find a net outflow of magnetic enthalpy through the lateral boundaries of the computational volume at the height of the jet front, with peak rates of about 0.15 in the $2.5 \mathrm{D}$ case and 0.05 in the $3 \mathrm{D}$ case. The outflow is transient in the $2.5 \mathrm{D}$ case, vanishing quickly after the jet front leaves the computational volume. In the 3D case, however, it turns into an inflow of the order -0.05 which persists until the end of the simulation. The energy in the radial magnetic field increases correspondingly, mainly outside the jet at $\vartheta>5.7^{\circ}$.

\subsection{Magnetic field: poloidal vs. toroidal}

The red line in Fig. 6 shows the magnetic flux $\Xi$ contained within an angle $\vartheta<5.7^{\circ}$ from the axis,

$\Xi(r, t):=\int_{\substack{r=\text { const. } \\ \vartheta<5.7^{\circ}}} B_{r}(t) \mathrm{d} A$,

divided by its initial value. For comparison, the green curve shows $\Xi^{+}$, the flux of only those field lines that have the same direction as the initial field (the green and the red curves coincide in the $2.5 \mathrm{D}$ case). Although the $2.5 \mathrm{D}$ jet fills only part of the $5.7^{\circ}$ cone, it causes a significant reduction of $\Xi$. This reflects the lateral expansion of the jet due to the pressure exerted by $B_{\varphi}$. There is less reduction in the $3 \mathrm{D}$ case, with $\Xi$ being near the original value at very large distances. The difference becomes evident where the $3 \mathrm{D}$ jet is unstable, showing that it is due to the dissipation of the toroidal field. There is only a small difference between the red and green curve in the $3 \mathrm{D}$ case, meaning that negative values of $B_{r}$ contribute little to the net flux. This shows 


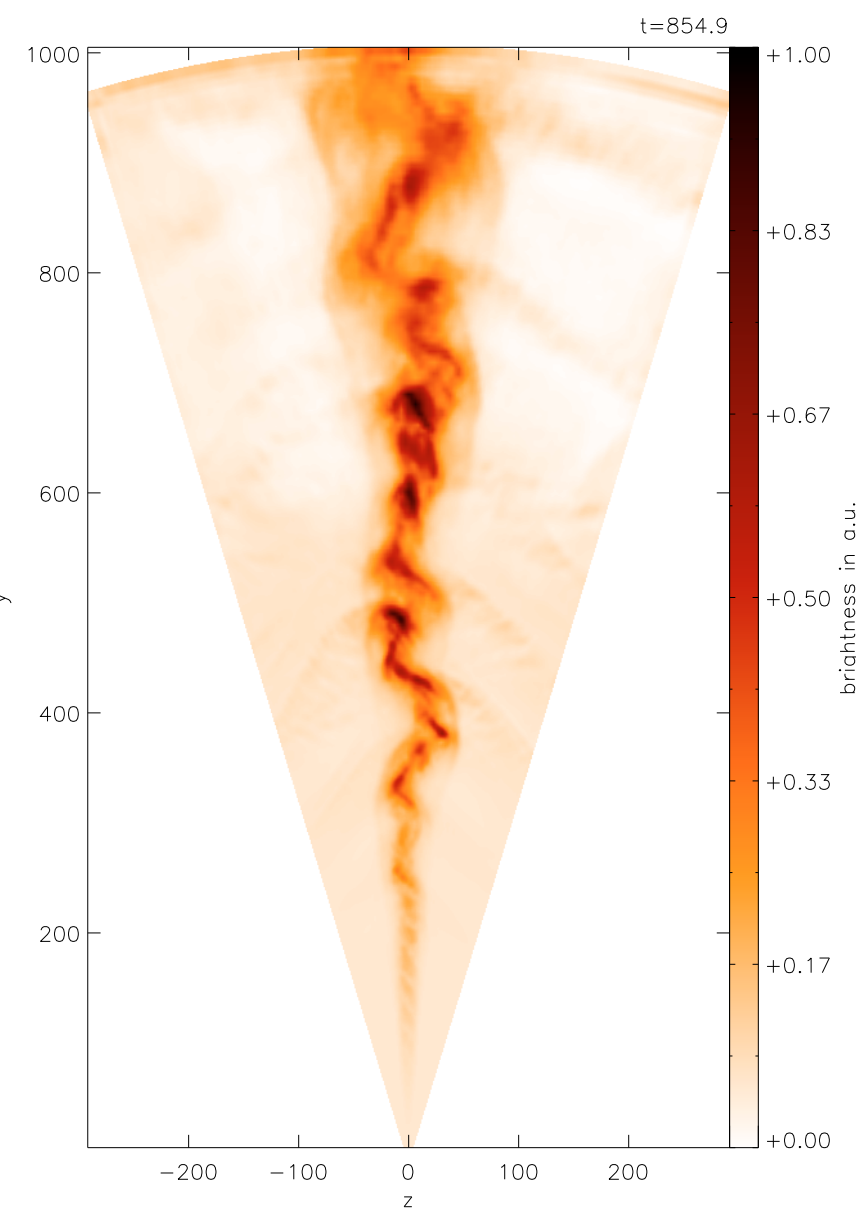

Fig. 2. Volume rendered image of the 3D jet that shows what it might look like in observations. For the volume rendering, a simple model was used in which emissivity and opacity depend on temperature and magnetic field strength. The jet exhibits a wiggly structure with bright knots, produced by the instabilities and the dissipation of magnetic energy.

that the toroidal field component dissipates without producing much "tangling" of the field lines.

Figure 7 compares the mean energy in the poloidal and toroidal magnetic fields over the width of the jet as a function of distance, the width of the jet being defined by a suitable velocity threshold. The 3D and 2.5D jets start out similar, with the mean poloidal and toroidal fields becoming comparable near the Alfvén surface. Beyond that distance, the mean toroidal field energy increases more strongly in the $2.5 \mathrm{D}$ case, roughly proportional to $r^{-2}$. In the 3D case, the instability-induced destruction of the toroidal field causes the slope to steepen substantially at $r \gtrsim 200$, approximately as $\left\langle B_{\varphi}^{2}\right\rangle \sim r^{-3}$. The mean poloidal field energy, on the other hand, stays near the initial value in the $3 \mathrm{D}$ case while decreasing somewhat in the $2.5 \mathrm{D}$ case. Taking the ratio between the energies, we find the magnetic field to be predominantly toroidal in the $2.5 \mathrm{D}$ case and predominantly poloidal in the 3D case at large distances.

We also experimented with means of the form $\int X v_{r} \mathrm{~d} A / \int v_{r} \mathrm{~d} A$, where the integral is performed over the whole $r=$ const. surface, and obtained similar results. Taking the mean within the static $\vartheta<5.7^{\circ}$ cone instead of using a velocity threshold yields a smaller value for $\left\langle B_{\varphi}^{2}\right\rangle /\left\langle B_{\mathrm{p}}^{2}\right\rangle$ in the 2.5D case, viz. $2 \pm 0.2$ for $r>200$. This is because an angle of $5.7^{\circ}$ includes more of the environment of the jet (cf. Fig. 4).

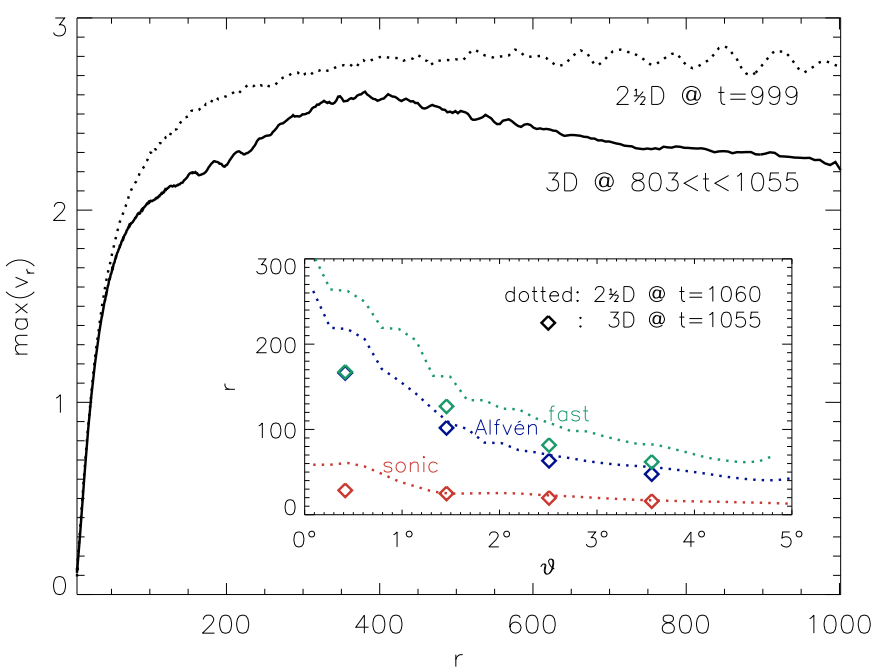

Fig. 3. Maximum velocity in jet direction as a function of distance. The inset shows the location of the critical surfaces. The flow passes first the sonic, then the Alfvén and finally the fast magnetosonic surface.

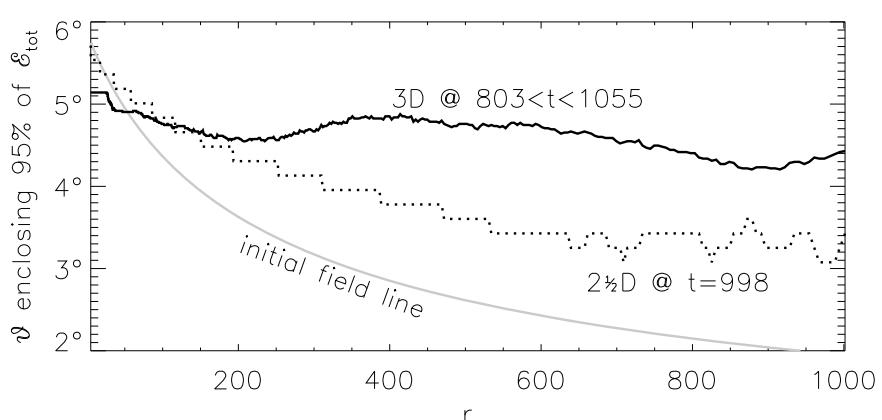

Fig. 4. Jet boundary, defined by the angle that encloses $95 \%$ of the total energy flow (see Eq. (8)), as a function of distance. The unstable 3D jet is less collimated than the $2.5 \mathrm{D}$ jet.

In a ballistically expanding jet (constant velocity and opening angle) the magnetic field components vary with distance $r$ as $B_{r} \sim r^{-2}, B_{\theta} \sim B_{\phi} \sim r^{-1}$. Integrals over the width of the jet of $B_{r}, B_{\phi}^{2}$ and $B_{\theta}^{2}$ are then constants. These integrals are shown in Fig. 8. The ballistic approximation works well in the $2.5 \mathrm{D}$ case, for $B_{r}$ as well as for $B_{\varphi}$, if the acceleration region is excluded. In the 3D case however, the integral of $B_{r}$ increases by about an order of magnitude along the jet, while the integral of $B_{\varphi}^{2}$ decreases over the entire range.

Figure 9 shows some poloidal magnetic field lines and the radial velocity in the $2.5 \mathrm{D}$ and $3 \mathrm{D}$ jet. The latter was "axisymmetrized" by averaging over the azimuthal coordinate $\varphi$. In the outer, high- $\vartheta$, part of the $2.5 \mathrm{D}$ jet, where the toroidal field is especially strong and jet acceleration most efficient, there is an increase of the angular separation between the field lines which is absent in the $3 \mathrm{D}$ jet. In other words, the poloidal magnetic flux decreases locally faster with distance in the $2.5 \mathrm{D}$ case.

\subsection{Forces and powers}

To identify the accelerating forces that generate the kinetic energy flow discussed in Sect. 3.2, we compute $P=\int \boldsymbol{F} \cdot \boldsymbol{v} \mathrm{d} V$, the instantaneous power (rate of work) delivered by a specific force $\boldsymbol{F}$ in the direction of the flow in the integrated volume. The combination of gas pressure and gravitational forces, $-\nabla p-\rho \boldsymbol{\nabla} \Phi$, accounts for about one third of the power delivered by the sum 

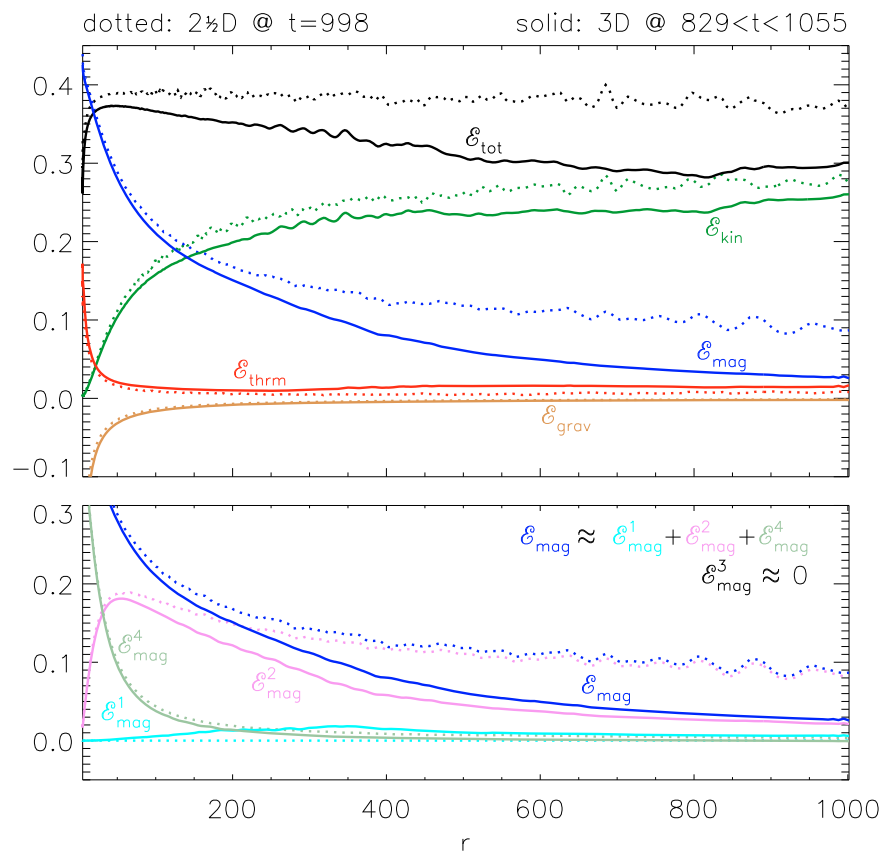

Fig. 5. Energy flow rates through the $r=$ const. surface. Magnetic enthalpy (integral over Poynting flux, blue line) is reduced in the $3 \mathrm{D}$ simulation as $B_{\varphi}^{2}$ decays as a consequence of instabilities.

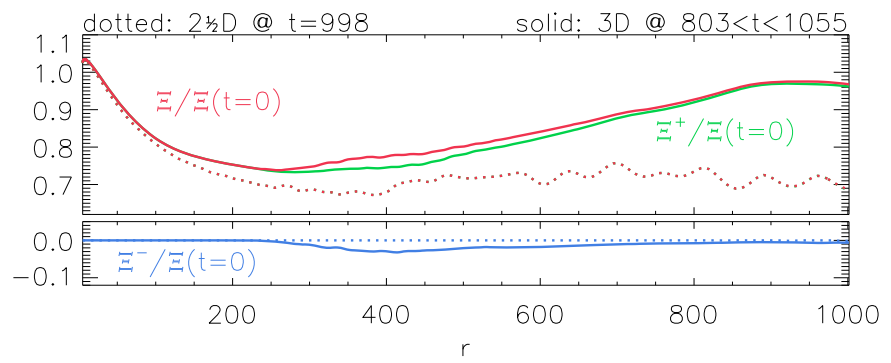

Fig. 6. Red: magnetic flux $\Xi(r)$ within $5.7^{\circ}$ from the jet axis, normalized by its initial value. Green: flux of $B_{r}^{+}>0\left(B=B_{r}^{+}+B_{r}^{-}\right)$, see Sect. 3.3.

of all forces in the whole volume, the corresponding acceleration takes place mainly below $r \approx 30$ (sonic surface). The rest is accounted for by the Lorentz force, which we decompose as follows:

$$
\begin{aligned}
& \frac{1}{4 \pi}(\boldsymbol{\nabla} \times \boldsymbol{B}) \times \boldsymbol{B}=\frac{1}{4 \pi}[\left(\boldsymbol{\nabla} \times \boldsymbol{B}_{\mathrm{p}}\right) \times \boldsymbol{B}_{\mathrm{p}}+\left(\boldsymbol{\nabla} \times \boldsymbol{B}_{\varphi}\right) \times \boldsymbol{B}_{\varphi} \\
&\left.+\left(\boldsymbol{\nabla} \times \boldsymbol{B}_{\mathrm{p}}\right) \times \boldsymbol{B}_{\varphi}+\left(\boldsymbol{\nabla} \times \boldsymbol{B}_{\varphi}\right) \times \boldsymbol{B}_{\mathrm{p}}\right],
\end{aligned}
$$

with $\boldsymbol{B}_{\varphi}=B_{\varphi} \hat{\boldsymbol{e}}_{\varphi}$ and $\boldsymbol{B}_{\mathrm{p}}=\boldsymbol{B}-\boldsymbol{B}_{\varphi}$. The corresponding components of $P$ are plotted in Fig. 10 as a function of the upper integral limit.

The last force in Eq. (11) has only an azimuthal component. It is important mainly below the Alfvén radius $(r \sim 60)$, exerting a torque in the same direction in which rotation is applied at the lower boundary. The next-to-last force vanishes in the axisymmetric case. In the general case, it has only non-azimuthal components. Unlike the last force, it works against the flow; the two forces largely cancel each other in the 3D simulation. The Lorentz force associated with $B_{\varphi}$ [second term in Eq. (11)] performs the same work in the 3D and 2.5D cases, despite the steepening of the toroidal magnetic pressure profile caused by the dissipation. The total power is also similar in the two cases,

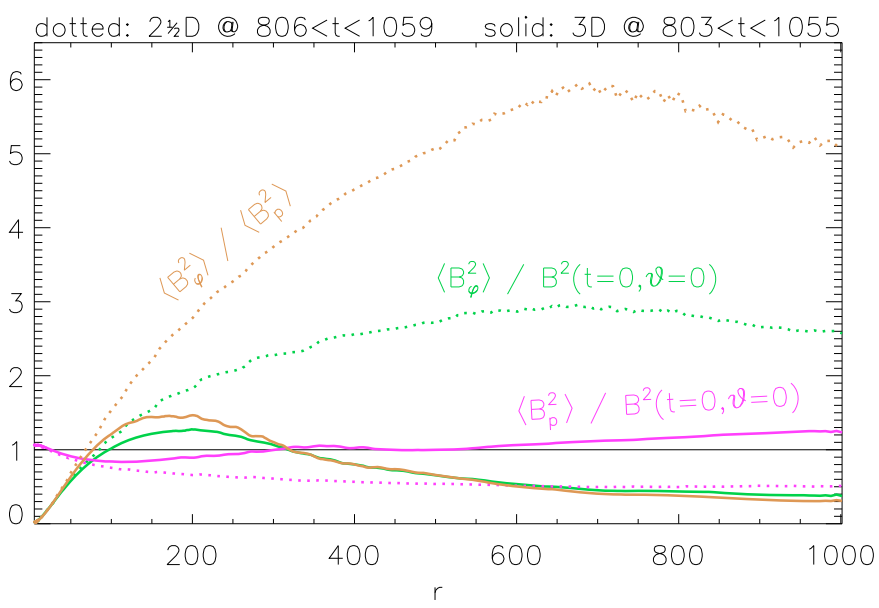

Fig. 7. Mean magnetic energies in the poloidal and toroidal fields within the jet, normalized by the initial on-axis magnetic energy, and their ratio. Without instability (2.5D), the field becomes predominantly toroidal at large distances. With instability (3D), it becomes predominantly poloidal.

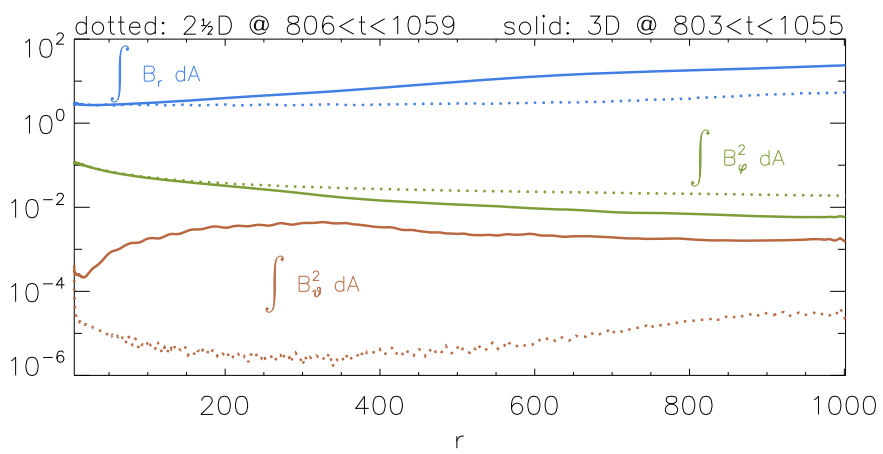

Fig. 8. Magnetic field components, integrated over the jet cross section, as a function of distance, on a logarithmic scale. In a spherically expanding ballistic flow these quantities would be constant, see text.

in agreement with the similarity of the kinetic energy flows in Fig. 5.

The situation is a bit different if only the radial power $\int F_{r} v_{r} \mathrm{~d} V$ is taken into account. The Lorentz force associated with $B_{\varphi}$ does approximately $20 \%$ more work in the whole volume in the $3 \mathrm{D}$ case, the additional power is delivered above $r \gtrsim 200$. The power of the net force, however, is approximately the same in both cases.

\section{Summary and discussion}

We have simulated jets generated by twisting a parabolically shaped large-scale magnetic field in both 3D and axisymmetric 2.5D. The shape of the initial field reflects itself in a fair amount of jet collimation, with an opening angle that decreases with distance, thus facilitating the growth of instabilities. The simulations cover the acceleration phase, where the jet passes through the critical surfaces (sonic, Alfvén and fast magnetosonic) for stationary MHD flows, as well as a substantial distance beyond these. We thus observe the onset of kink instabilities above the Alfvén surface in the 3D simulation. The instabilities disrupt the magnetic field structure. They cause magnetic dissipation, significantly reducing the toroidal field strength and with it the flow rate of magnetic enthalpy (surface integral of the Poynting flux) 

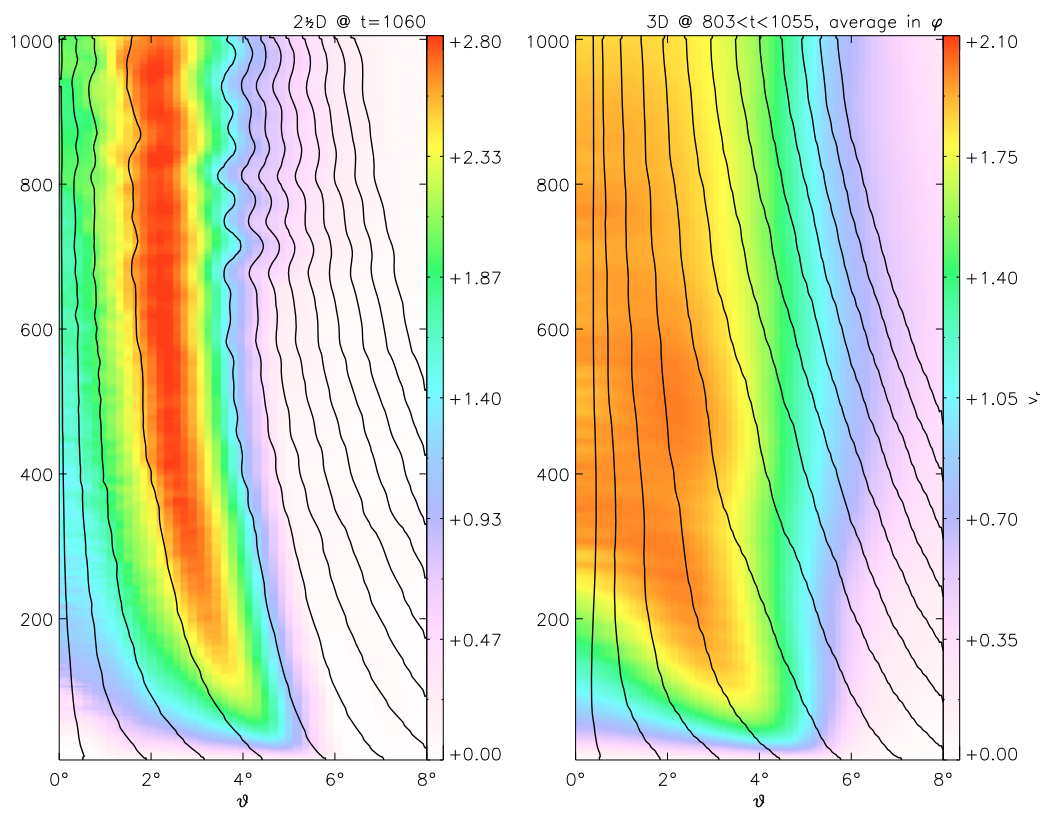

Fig. 9. Poloidal magnetic field lines and radial velocity field, plotted as a function of direction $\vartheta$ (horizontal axis) and distance $r$ (vertical axis); a conical magnetic surface would appear as a vertical line. The left-hand plot shows a snapshot of the $2.5 \mathrm{D}$ case, the right-hand plot shows the $3 \mathrm{D}$ case with the magnetic field and velocity being averaged in azimuthal direction as well as time. Both plots show the same field lines in the sense that they start from the same direction at the lower boundary. The angular separation between field lines in the outer, high- $\vartheta$ part of the jet (here: between the 4th and 5th line from the left) increases noticeably in the $2.5 \mathrm{D}$ case, leading to acceleration by the magnetic "diverging nozzle" effect there, see discussion.

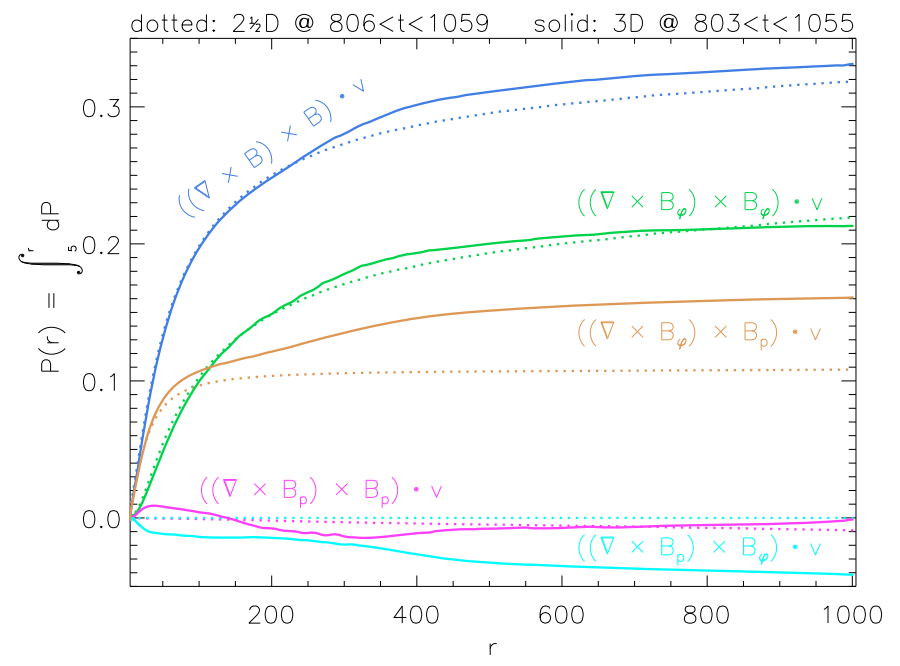

Fig. 10. Rate of work done in the volume delimited by the lower boundary and $r$ by the components of the Lorentz force in the direction of the flow. The power delivered by the total Lorentz force is about the same in the $3 \mathrm{D}$ and $2.5 \mathrm{D}$ cases.

on a length scale of about 2-15 times the minimal Alfvén distance in the jet.

A direct comparison of the 2.5D and 3D simulations reveals no significant difference in the way the kinetic energy of the jet (integrated over its cross section) increases with distance. However, the distribution of the kinetic energy across the jet indicates differences in the acceleration process (cf. Fig. 9). In the axisymmetric flow, the acceleration is restricted to magnetic surfaces that diverge from each other more rapidly than in a flow with fixed opening angles. This creates a "magnetic nozzle" effect (Begelman \& Li 1994) restricted to a limited range of angles within the flow (cf. discussion in Spruit 2008). A similar case of non-uniform expansion has been found in relativistic flows by Tchekhovskoy et al. (2008). In the 3D case, the (time averaged) acceleration is more uniform across the jet. It thus seems that while the steepening of the magnetic pressure gradient caused by the dissipation of the toroidal field leads to additional acceleration in the $3 \mathrm{D}$ case, this is made up for by a more favorably distributed poloidal magnetic flux in the $2.5 \mathrm{D}$ case which also enhances acceleration. In other words, there are different mechanism at work which yield the same result. Whether it is coincidence that the two effects have nearly the same strength is not clear.

The energy released by the dissipation of the toroidal magnetic field may be radiated as light. The yield depends on the details of the dissipation (reconnection) and radiation mechanisms involved. Since the magnetic energy density $\left(B_{\varphi}^{2} / 8 \pi\right)$ accounts for half of the Poynting flux $\left(B_{\varphi}^{2} v_{r} / 4 \pi\right)$, the present simulations suggest that the available luminosity may be as much as $10 \%$ of the initial magnetic enthalpy flow rate (Fig. 5), provided that half of it is converted into kinetic energy and the rest into light. As dissipation is not a smooth process, the emission will be stronger in some regions. These would likely turn up as bright knots in observations. Together with the wiggles caused by the kink instabilities, the knots produce a structured appearance that is similar to what has been observed in protostellar jets (e.g. Heathcote et al. 1996; Reipurth \& Bally 2001). The knots in the mocked jet image move with the flow, consistent with observational findings (Eislöffel \& Mundt 1992; Hartigan et al. 2005). This provides an alternative to the "internal shock" interpretation usually invoked to explain jet knots (e.g. Hartigan \& Raymond 1993).

The magnetic structure of a jet undergoes a dramatic change if it becomes subject to violent kink instabilities. The formerly ordered helical structure is largely destroyed and the poloidal field becomes the dominating field component. However, magnetic flux conservation still implies that the poloidal field declines faster with distance than the toroidal one. The toroidal field may thus, beyond the region covered by the here-presented simulations, become again dominant. This could, in principle, lead to a resurgence of instabilities until the Alfvén speed has dropped below the sideways expansion speed of the jet (times a factor of order unity). From this point on the toroidal field is effectively frozen in the flow (cf. discussion in Paper I).

\subsection{Collimation and jet environment}

Collimation of jets is popularly attributed to the "hoop stress" in the toroidal field component. This is misleading: though the stress contributed by the toroidal field can compress the configuration near the axis (as observed in simulations), it eventually 
has to be taken up by an external agent (for a more extended discussion, see Spruit 2008). In the simulations presented above, as well as in other work, this agent is an external medium surrounding the jet. It is included mostly because of limitations of the codes used, since the demands of conserving energy typically cause numerical instabilities at low gas densities or low plasma- $\beta$.

The boundary between the jet and the external medium actually expands due to pressure exerted by the toroidal field. The role of a (material) external medium in confining the jet can be taken over by a magnetic field, if it is able to transfer stress in the jet's toroidal field to the surface of the accretion disk. One might imagine that a toroidal field extending around the jet might serve this role. The high Alfvén speeds in this field, however, would make it violently unstable to non-axisymmetric instabilities, as the early history of magnetic configurations for controlled fusion (linear pinches) testifies. This obstacle does not become evident in the axisymmetric models in the literature. A more realistic possibility, proposed by Shu et al. (1995), is that the disk's poloidal field, assumed for launching the jet in the inner regions, actually extends to much larger distances in the disk. Deformation of the field can take up the lateral stress exerted by the jet. The collimation of the jet would then be directly related to the properties of this poloidal field. High degrees of collimation would be most easily achieved in disks with a large ratio of outer to inner disk radius (Spruit et al. 1997). Numerical simulations at much lower plasma- $\beta$ than currently feasible would be needed to study this form of "poloidal collimation".

As pointed out in the introduction, instabilities may take some distance to travel to become effective. The results presented above show that a distance of the order of 1000 times the source size of the jet is needed to capture the dissipation of the toroidal field. I surmise this to be the reason why the effect of instabilities is not as noticeable in the works of Anderson et al. (2006) and Ouyed et al. (2003), rather than the lack of external confinement of these winds.

\subsection{Disruption}

The possible presence of instabilities in jets sometimes raises concern about disruption. A complete dissipation of the jet into its surroundings is possible in principle through instabilities driven by the interaction of the jet with its surroundings, for example by Kelvin-Helmholtz instabilities, or its termination in a "hot spot". These processes extract their energy directly from the bulk kinetic energy of the flow. The effect of internal instabilities deriving from the free energy in the toroidal field is much less destructive, since these mainly redistribute internal energy forms within the jet. The comparison between the $2.5 \mathrm{D}$ and the 3D cases shows that the 3D jet widens by less than a factor 2 as a result of internal instabilities (cf. Figs. 4 and 9). The non-axisymmetric nature of the instabilities thus does cause some interaction with the environment, but its consequences remain relatively benign.

\subsection{Cold flows}

Current 3D simulations are poorly equipped to handle flows in which the magnetic or kinetic energy density, or both, dominate over the thermal energy density. In magnetically dominated flows driven from actual accretion disks, the temperature of the plasma is often sufficiently low that magnetic energy density dominates over plasma pressure already at a short distance from the disk surface (Blandford \& Payne 1982). As a consequence, the sonic point in the present results is further away from the source than would be the case in e.g. actual protostellar disks. It is possible that the onset of the instabilities in more strongly magnetically dominated flows would be faster, and their consequences even stronger than in the simulations presented here. Codes specially designed to handle such "cold" flows would be needed to verify this.

Acknowledgements. The author thanks H. C. Spruit for fruitful discussions and a critical reading of the manuscript, and M. Obergaulinger for providing his MHD code.

\section{References}

Anderson, J. M., Li, Z.-Y., Krasnopolsky, R., \& Blandford, R. D. 2006, ApJ, 653, L33

Begelman, M. C., \& Li, Z.-Y. 1994, ApJ, 426, 269

Blandford, R. D., \& Payne, D. G. 1982, MNRAS, 199, 883

Cao, X., \& Spruit, H. C. 1994, A\&A, 287, 80

Drenkhahn, G. 2002, A\&A, 387, 714

Eislöffel, J., \& Mundt, R. 1992, A\&A, 263, 292

Giannios, D., \& Spruit, H. C. 2006, A\&A, 450, 887

Hartigan, P., \& Raymond, J. 1993, ApJ, 409, 705

Hartigan, P., Heathcote, S., Morse, J. A., Reipurth, B., \& Bally, J. 2005, AJ, 130, 2197

Heathcote, S., Morse, J., Hartigan, P., et al. 1996, AJ, 112, 1141

Junor, W., Biretta, J. A., \& Livio, M. 1999, Nature, 401, 891

Königl, A., \& Choudhuri, A. R. 1985, ApJ, 289, 173

Moll, R., Spruit, H. C., \& Obergaulinger, M. 2008, A\&A, 492, 621

Mundt, R., Buehrke, T., Solf, J., Ray, T. P., \& Raga, A. C. 1990, A\&A, 232, 37

Obergaulinger, M. 2008, Ph.D. Thesis, Max-Planck-Institut für Astrophysik, Garching bei München

Ouyed, R., Clarke, D. A., \& Pudritz, R. E. 2003, ApJ, 582, 292

Reipurth, B., \& Bally, J. 2001, ARA\&A, 39, 403

Shu, F. H., Najita, J., Ostriker, E. C., \& Shang, H. 1995, ApJ, 455, L155

Sikora, M., Begelman, M. C., Madejski, G. M., \& Lasota, J.-P. 2005, ApJ, 625, 72

Spruit, H. C. 2008, [arXiv:0804 . 3096]

Spruit, H. C., Foglizzo, T., \& Stehle, R. 1997, MNRAS, 288, 333

Tchekhovskoy, A., McKinney, J. C., \& Narayan, R. 2008, MNRAS, 388, 551

Todo, Y., Uchida, Y., Sato, T., \& Rosner, R. 1993, ApJ, 403, 164 